\title{
Quality Analyses of Fine Structures Transfer in the Photorealistic Images
}

\author{
S. V. Sai, I. S. Sai, N. Yu. Sorokin \\ Department of automation and information technologies, Pacific National University, Khabarovsk, Russia \\ Email: sai@evm.khstu.ru,nus@mail.khstu.ru
}

Received 2012

\begin{abstract}
This work presents method of the quality analyses of the photorealistic images. Developed method utilizes proposed criteria of image definition quality for fine details. Presented method has the following significant property: the quality estimation is provided without test images or patterns. Algorithm for search and recognition of fine structures in the photorealistic images using the predefined criterion is considered.
\end{abstract}

Keywords: Image Quality Analyses; Search Algorithm; Fine Structures Recognition

\section{Introduction}

Quality control of the compressed digital image is complex and ambiguous. Usually the distortion definitions of the static and dynamic test signals are the indirect values of the quality estimation of the broadband digital system. However, systems of the compressed television signals have to be analyzed using more complex parameter measurements. Image quality in such systems changes dynamically depending on the data rate, complexity of the transferred image and applied coding algorithm. Static test signals cannot present real quality characteristics of the image. More complex analyses instead of simple test signals must be carried out, i.e. natural test images in compressed mode must be used.

Objective analyses of the digital video signals can be produced using the current measurement systems (like PQA200, PQA300, PQA500, etc.). In general, these types of equipment are used by the vendors of the video compression systems for the quality analyses of codecs. But the application of such equipment is not suitable when the multimedia system has been already selected by the broadcasting company using some criteria.

Suppliers of the multimedia services provide highly compressed images in a low quality pack because of absence of the standard methods of quality analyses for digital images and video. In most cases user has no original image to compare with, thus he estimates the "good" or "bad" quality of the received image using his experience of visual perception of such properties as image definition, sharpness, contrast, saturation, presence of the artifacts. Modern analyzers use the quality analyses methods that are based on comparison of the differences between test and distorted (corrupted) images [1-6]. This comparison is carried out for some selected visual model. Thus, the absence of test images does not allow the application of such analyzers.

Therefore, finding new methods of the quality analyses is an actual problem. Such methods must provide an objective estimation of image definition quality and sharpness degradation without the test patterns.

This article proposes the method of the visual image definition quality estimation of the photorealistic image using the objective criterion without the test image. Special features of the proposed algorithm for search and recognition of fine structures in the real images are considered.

\section{Algorithm for search and recognition}

In order to estimate the image definition quality search and recognition of the fine structures must be carried out. Such structures include fine details like dot objects or thin line fragments with the size from one to several pixels.

Search algorithm consists of the following stages. In the first stage the transformation of the primary color signals (RGB) to the equal color space $W^{*} U^{*} V^{*}$ is produced for each pixel [7] using the following equation:

$$
\begin{aligned}
& W^{*}=25 Y^{1 / 3}-17, \\
& U^{*}=13 W^{*}\left(u-u_{o}\right), \\
& V^{*}=13 W^{*}\left(v-v_{o}\right),
\end{aligned}
$$

where $Y$ is the luminance, changed from 1 to 100 , $W^{*}-$ is the brightness index; $U^{*}$ and $V^{*}-$ are the chromaticity indices; $u$ and $v$ - are the chromaticity 
coordinates in Mac-Adam diagram [8]; $u_{o}$ and $v_{o}-$ are the chromaticity coordinates of basic white color with $u_{o}=0,201, v_{o}=0,307$.

Equal color spaces $\left(W^{*} U^{*} V^{*}, L^{*} a^{*} v^{*}\right.$, etc.) are used traditionally for the estimation of color transfer quality for the big details. Here estimation of the color differences $\Delta$ is computed as

$$
\Delta=3 \sqrt{\left(\Delta W^{*}\right)^{2}+\left(\Delta U^{*}\right)^{2}+\left(\Delta V^{*}\right)^{2}}
$$

where $\quad \Delta W^{*}=W_{o}^{*}-\tilde{W}_{o}^{*} ; \quad \Delta U^{*}=U_{o}^{*}-\widetilde{U}_{o}^{*} ;$ $\Delta V^{*}=V_{o}^{*}-\widetilde{V}_{o}^{*} ; W_{o}^{*} U_{o}^{*} V_{o}^{*}-$ are the color coordinates of the big detail from the test image; $\widetilde{W}_{o}^{*} \widetilde{U}_{o}^{*} \widetilde{V}_{o}^{*}-$ are the color coordinates of the distorted image. The color transfer quality is determined by the number of the minimum perceptible color difference (MPCD). If $\Delta<1$ then the color differences are invisible for the human eye.

In our works [9-10] we proposed method of the color differences estimation between the fine detail $\left(W_{o}^{*} U_{o}^{*} V_{o}^{*}\right)$ and the color coordinates of the background pixels ( $\left.W_{b}^{*} U_{b}^{*} V_{b}^{*}\right)$ using the normalized equal color space. The following criterion was used:

$$
\Delta K=\sqrt{\left(\frac{W_{o}^{*}-W_{b}^{*}}{\Delta W_{t h}^{*}}\right)^{2}+\left(\frac{U_{o}^{*}-U_{b}^{*}}{\Delta U_{t h}^{*}}\right)^{2}+\left(\frac{V_{o}^{*}-V_{b}^{*}}{\Delta V_{t h}^{*}}\right)^{2}},
$$

where $\Delta K$ - color contrast of the fine detail relatively to background; $\Delta W_{t h}^{*}, \Delta U_{t h}^{*}, \Delta V_{t h}^{*}$ - are the thresholds according to brightness and chromaticity indices for fine detail. Threshold values on brightness and chromaticity indices depend on the size of fine detail, background color coordinates, time period of object presentation and noise level. For fine details with sizes not exceeding one pixel the threshold values are obtained experimentally. In particular [9], for fine details of the test pattern located on a grey background $\left(70<W_{b}^{*}<90\right)$ threshold values are approximately $\Delta W_{t h}^{*} \approx 6 \quad$ MPCD and $\Delta U_{t h}^{*} \approx \Delta U_{t h}^{*} \approx 72$ MPCD.

In the second stage the criterion (2) is used for the estimation of quality and recognition of fine details. For this, the processed image is scanned with the $3 \times 3$ pixels window. Recognition algorithm of the fine structure is executed for each iteration step.

During the analyses we should recognize an object: is it a dot of a thin line. For this we use the binary images of the fine structures presented on Figure 1. Using images we can obtain the spatial coordinates of the object and background.
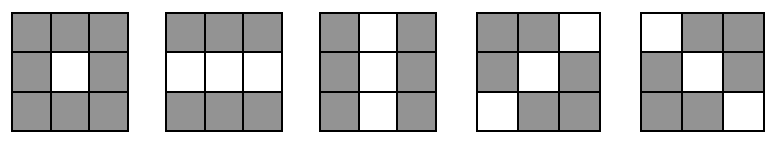

Figure 1. Binary images of the fine structures.
Analyses are started with the first structure - a dot (left image on Figure 1). On the first step the mean values of the color coordinates of the background $\left(\bar{W}_{b}^{*} \bar{U}_{b}^{*} \bar{V}_{b}^{*}\right)$ and object $\left(\bar{W}_{o}^{*} \bar{U}_{o}^{*} \bar{V}_{o}^{*}\right)$ are computed.

Next, the following condition is checked:

$$
\Delta_{b}=\frac{1}{N} \sum_{n=1}^{N} \Delta K_{n}<0,5
$$

where $n-$ is a relative number of the background pixel in the current window $(3 \times 3) ; N-$ is a total number of background pixels in the window; $\Delta K_{n}-$ is a color contrast of the pixel relative to mean value of the background color, computed as

$$
\Delta K_{n}=\sqrt{\left(\frac{W_{n}^{*}-\bar{W}_{b}^{*}}{\Delta W_{t h}^{*}}\right)^{2}+\left(\frac{U_{n}^{*}-\bar{U}_{b}^{*}}{\Delta U_{t h}^{*}}\right)^{2}+\left(\frac{V_{n}^{*}-\bar{V}_{b}^{*}}{\Delta V_{t h}^{*}}\right)^{2}} .
$$

Condition (3) means that the color differences between the background pixels are invisible for an eye. Similarly, the color differences for the object pixels are computed:

$$
\Delta_{o}=\frac{1}{M} \sum_{m=1}^{M} \Delta K_{m}<0,5,
$$

where $m-$ is a relative number of the object pixel; $M-$ is a total number of object pixels in a window $(3 \times 3)$; $\Delta K_{m}$ - color contrast of the object pixel relative to mean values $\left(\bar{W}_{o}^{*} \bar{U}_{o}^{*} \bar{V}_{o}^{*}\right)$, that are produced similar to (4).

If the conditions (3) and (5) are fulfilled, the contrast of the object relatively to the background is computed

$$
\Delta K_{o / b}=\sqrt{\left(\frac{\bar{W}_{o}^{*}-\bar{W}_{b}^{*}}{\Delta W_{t h}^{*}}\right)^{2}+\left(\frac{\bar{U}_{o}^{*}-\bar{U}_{b}^{*}}{\Delta U_{t h}^{*}}\right)^{2}+\left(\frac{\bar{V}_{o}^{*}-\bar{V}_{b}^{*}}{\Delta V_{t h}^{*}}\right)^{2}}
$$

and afterwards the following condition is checked

$$
\Delta K_{o / b} \geq 2 \text {. }
$$

If (7) is satisfied than the decision is made that the dot object is recognized and we save the spatial coordinates of the center of this object $(i, j)$. After this we move the window further by three pixels and analyze another block of the processed image.

If conditions (3), (5) and (7) are not fulfilled the next structure (a fragment of the horizontal thin line) is processed. Also, if conditions (3), (5) and (7) are not fulfilled for each structure we decide that the current window has no recognized objects and the window is shifted further by one element.

Hereby, the proposed algorithm allows for recognition and selection of the fine structures - dots and thin lines fragments, noticeable for an eye. Note that compared to the algorithm described in the work [10] we do not use the comparison of the binary blocks to the binary masks. This reduces the number of computation steps significantly.

Figure 2 presents an example of the algorithm output, 
where the fine structures are selected from the photorealistic image $(244 \times 225$ pixels $)$.

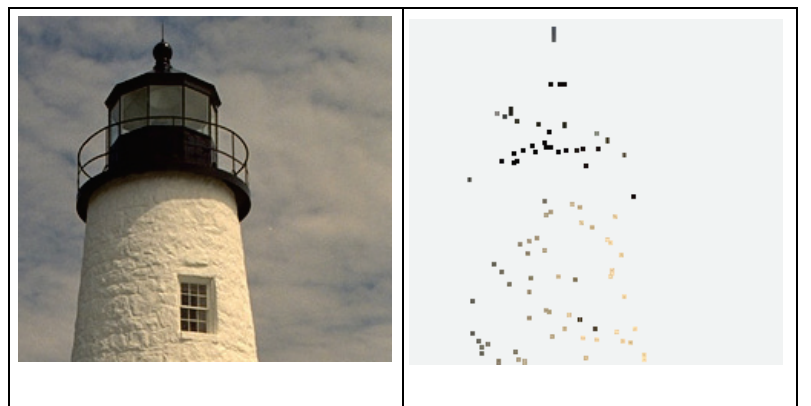

Figure 2. Example of the recognition of fine structures.

\section{Criterion of image definition quality}

Normally, the image definition quality is estimated using the resolution of the video system, i.e. by the number of reproduced pixels or the format of the image. For example, the format $1280 \times 960(1,23$ Megapixels $)$ means that the photo or video system is able to reproduce the fine details with sizes $1 / 1280$ and $1 / 960$ from width and height of the image frame, accordingly. So, the image will have a number of fine structures noticeable by an eye in case the image is not distorted. Particularly, for the image in Figure 2 number of recognized fine structures is equal to $N_{R}=0,18 \%$ from the total amount of pixels.

Obviously, that $N_{R}$ depends on the real number of fine details of the image and on the format of the image. However, the photorealistic image will always have some minimal value of $N_{R}$. This assumption is used in the developed method of the image definition quality estimation.

The method of the estimation consists of several stages. Assume that we have a number of photorealistic images provided by the multimedia service through the Internet. Usually, these images are transferred using the compressed format, like JPEG or JPEG-2000 standards. Each image $(m)$ can be presented in arbitrary format (number of pixels). For example, one of them can have $1280 \times 960$ pixels, another $800 \times 600$ pixels.

At the first stage, we process each image with the presented algorithm for search and recognition of the fine structures. We compute the mean value:

$$
\bar{N}_{R}=\frac{1}{M} \sum_{m=1}^{M} N_{R, m},
$$

where $M-$ is the number of processed images. This mean value is compared to the threshold $N_{T H}$

$$
\bar{N}_{R} \geq N_{T H} \text {. }
$$

If the criterion (9) is satisfied then the decision is made that the image definition quality corresponds to the presented format. This threshold value is selected experimentally after analyzing a big set of not distorted im- ages in different formats. As a result of the experiment we concluded that the image definition quality corresponds to the presented format in case the number of recognized fine structures is greater than the threshold $N_{T H}=$ $0,05 \%$.

If the criterion (9) is not satisfied, i.e. number of fine structures is less than $0,05 \%$, the decision is made that the image definition quality does not correspond to the presented format.

Nonfulfillment of the criterion (9) means that the processed images have lack of fine structures. This result can be explained by the following reasons: images were highly distorted due to the high level of compression in codec, or were obtained from the digital camera with the lower resolution than the image format.

As an example Figure 3 shows the result of image (from Figure 2) analyses after 2D Gaussian filtering. During the analyses the number of recognized fine structures is equal to $N_{R}=0,02 \%$ and it does not satisfy criterion (9).

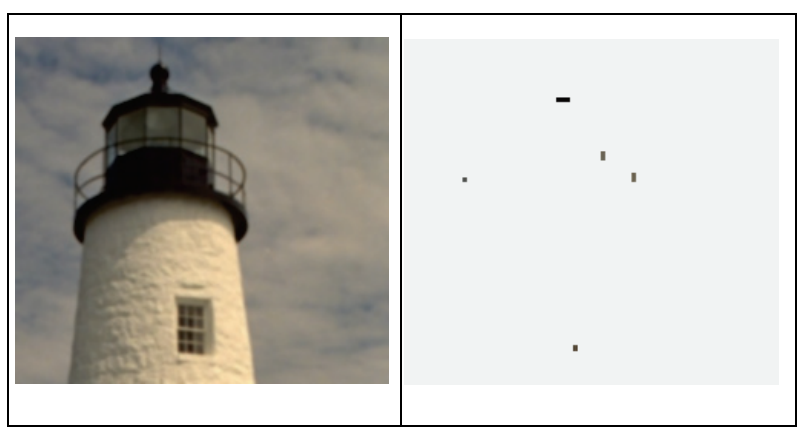

Figure 3. Analyses of the distorted image.

\section{Conclusion}

Let's emphasize the main features of the developed method of the analyses of the image definition quality for the photorealistic images compared to the known techniques.

Analyses are carried out based on the developed algorithm for search and recognition of the fine structures in images. Main property of the algorithm is that the recognition process is done using the MPCD of the fine details. Also the author's method of estimation of the color differences in the normalized equal color space is applied. The output from the algorithm contains the percent number of the recognized fine structures $\left(N_{R}\right)$ that are noticeable by an eye. The value $N_{R}$ is used in the proposed criterion (9) for the estimation of the real image definition quality of the photorealistic image.

Thus, compared to the known techniques our method does not require test images or patterns (containing fine structures, e.g., like dash lines), which is the main difference.

Developed criterion can be used for the video quality analyses. In his case, the same method should be applied 
to the series of video frames similarly to the photorealistic images. In this case, the estimation due to criterion (9) helps to decide if the image definition quality corresponds to the utilized video format.

\section{REFERENCES}

[1] Weisi Lin "Gauging Image and Video Quality in Industrial Applications", Studies in Computational Intelligence, 2008, Volume 116, Advances of Computational Intelligence in Industrial Systems, pp. 117-137. Doi: 10.1007/978-3-540-78297-1 6

[2] Wang, A. C. Bovik and L. Lu, "Why is image quality assessment so difficult?", IEEE International Conference on Acoustics, Speech, \& Signal Processing, May 13-17, 2002, Vol: 4, pp. 3313 -3316.

DOI: $10.1109 /$ ICASSP.2002.5745362

[3] Tektronix, Inc. Picture Quality Analysis System PQA300, http://www.tek.com, 2003.

[4] Tektronix, Inc. Picture Quality Analysis System PQA500, http://www.tek.com, 2010.

[5] Genista Corporation. Quality Assurance Solutions for IPTV and Mobile Media. http://www.genista.com, 2006.

[6] Opicom GmbH. Perceptual Voice, Audio and Video Quality. http://www.opticom.de, 2012.

[7] Wyszecki G. Uniform Color Scales: CIE $1964 \mathrm{U} * \mathrm{~V} * \mathrm{~W} *$ Conversion of OSA Committee Selection. In JOSA, Vol. 65, 1975, pp. 456-460.

[8] Mac Adam D.L. Uniform Color Scales / JOSA, Vol. 64. 1974, pp. 1691-1702.

[9] Sai S.V. "Methods of the Definition Analysis of Fine Details of Images," Chapter in the book: Vision Systems: Applications, G. Obinata and A. Dutta (eds.), I-Tech Education and Publishing, Vienna, Austria, 2007, pp. 279-296. DOI: $10.5772 / 4993$

[10] Sai S.V., N.Yu. Sorokin "Search Algorithm and the Distortion Analysis of Fine Details of Real Images" Pattern Recognition and Image Analysis. - 2009. - Vol. 19, № 2. - pp. 257-261. DOI: 10.1134/S1054661809020072 